\title{
Micro-Logistics Analysis for Human Space Exploration
}

\author{
Chel Stromgren ${ }^{1}$ and Ricardo Galan ${ }^{2}$ \\ Science Applications International Corporation, McLean, VA, 22102 \\ and \\ William Cirillo ${ }^{3}$ \\ NASA Langley Research Center, Hampton, VA, 23681
}

\begin{abstract}
Traditionally, logistics analysis for space missions has focused on the delivery of elements and goods to a destination. This type of logistics analysis can be referred to as "macro-logistics". While the delivery of goods is a critical component of mission analysis, it captures only a portion of the constraints that logistics planning may impose on a mission scenario. The other component of logistics analysis concerns the local handling of goods at the destination, including storage, usage, and disposal. This type of logistics analysis, referred to as "micro-logistics", may also be a primary driver in the viability of a human lunar exploration scenario. With the rigorous constraints that will be placed upon a human lunar outpost, it is necessary to accurately evaluate micro-logistics operations in order to develop exploration scenarios that will result in an acceptable level of system performance.
\end{abstract}

\section{Introduction}

Zor space missions that involve repeated or continuous human habitation, such as Mir, the International Space F Station (ISS), or the future lunar exploration scenario; logistics planning is a primary driver in overall system performance. Traditionally, the analysis of logistics has focused on the delivery of goods and elements to the destination $^{1,2}$. For the purposes of this paper, this type of logistics analysis will be referred to as "macro-logistics". While delivery constraints may be a driver in lunar exploration scenario performance, they are not the only aspect of logistics resupply that impose limitations on a scenario. The handling, usage, and disposal of goods at the destination can also be a major driver. This type of logistics analysis will be referred to as "micro-logistics".

Delivery and storage capabilities at a lunar outpost could be restrictive to such a degree that micro-logistics constraints could become a major driver in the operations of the outpost. These issues must be evaluated as an integral part of scenario analysis for the human lunar exploration scenario in order to develop elements designs, scenario descriptions, and operational plans that will result in an acceptable level of system performance.

This paper will present an overview of the micro-logistics analysis capability demonstrated for a NASA Constellation Program's human lunar outpost scenario analysis. In particular, the discussion will include the challenges presented by micro-logistics issues; the necessity of analyzing scenario performance based on micrologistics constraints; a description of the micro-logistics models that were developed for this analysis; and results of example micro-logistics analysis.

\section{Human Lunar Scenario Constraints}

Experience with previous and current extended human scenarios, such as Mir and ISS, has demonstrated that stowage, usage, and disposal of logistics can present significant challenges. These types of challenges are likely to be even more rigorous in a human lunar exploration scenario, where certain constraints will be even more severe.

The total pressurized volume of a human lunar outpost is expected to be much less than is available in the low Earth orbit (LEO) space stations, especially for early missions. Therefore, the volume available for stowage of logistics will be proportionally smaller. In addition, a significant fraction of the habitable volume that is available will be initially used for the transportation of logistics to the surface, opening up only as the goods are consumed. The net result is that there will be a limited capability to stow goods and trash within the habitat elements.

${ }^{1}$ Chief Scientist, Modeling and Analysis Division, 1710 SAIC Drive, AIAA Member.

${ }^{2}$ Systems Engineer, Modeling and Analysis Division, 1710 SAIC Drive.

${ }^{3}$ Senior Researcher, Space Mission Analysis Branch, MS 462, AIAA Member. 
The transportation schedule and capability are also likely to be more constrained for a human lunar outpost. The total quantity of goods delivered to the surface per year will be relatively small, as compared to the capacity that can be provided to LEO. In addition, the delivery of goods will not happen in conjunction with the crew and will occur in relatively large increments. Because the lunar outpost scenarios rely on the use of a cargo lander, in conjunction with a crewed lander with limited delivery capacity, most goods will be delivered ahead of time and stockpiled for usage. It is possible that a single cargo lander might contain goods to be used by multiple future crews. Therefore, goods might be required to remain on the surface, within a carrier, for an extended period of time.

The schedule for deliveries to the lunar outpost will also be constrained. Unlike the ISS, where the Space Shuttle, Progress, Automated Transfer Vehicle (ATV), and eventually H-II Transfer Vehicle (HTV) provide delivery of logistics at multiple opportunities throughout the year, only the cargo lander will provide significant delivery capability to the lunar surface. Therefore, it is expected that there will only be one or two possible opportunities to deliver cargo per year to the lunar outpost.

Finally, the ability to dispose of trash and used goods will be basically non-existent at the lunar outpost site. The crewed lander will provide an extremely limited cargo return capability to Earth, but other high priority cargoes will most likely consume that available volume. Unlike the ISS, there is no ability to load up exhausted delivery vehicles and destructively dispose of them. Therefore, all trash and used goods will have to be permanently stored at the outpost site.

\section{Micro Logistics Challenges}

The constraints listed above, all of which are related to micro-logistics, have the potential to impose severe restrictions on the operations of the lunar outpost. Initial subjective analysis of potential lunar outpost scenarios led to several specific concerns regarding micro-logistics operations:

- Storage volume required within the habitat - A primary concern with the human lunar outpost is the total volume that will be required in the habitat to store goods. Because there is a minimum volume required for human habitation, any additional volume required for storage will increase the total required size of the habitat. A larger volume will require larger elements with a greater mass, which could in turn, lengthen the time required to build-up the outpost, reducing the overall productivity. A more accurate, dynamic analysis of storage requirements is needed to determine exactly how large the outpost habitat elements will need to be to support logistics storage.

- Required storage capacity for gasses and liquids - It is anticipated that the outpost will use some degree of closure in the Environmental Control and Life Support System (ECLSS), in order to reduce the total volume of gasses and liquids that must be delivered. Because the delivery, usage, and processing of gasses and liquids in the ECLSS system is stochastic, there will be requirements to store gasses and liquids in various forms for varying periods of time. A dynamic analysis of ECLSS system operations is needed to evaluate storage capacity requirements to better inform the design of these systems.

- Movement and manipulation of carriers on the surface - The resupply of logistics goods for the human lunar outpost will rely on the use of carriers delivered by a cargo lander. For gasses and liquids, carriers will take the form of tanks that will have to be unloaded from the lander, moved to the habitat, and connected to the ECLSS system. For pressurized goods, carriers will be pressurized and conditioned vessels that will be unloaded, transported, and connected to the crew habitat. The crew will enter the carriers to unload goods. Expended carriers will eventually be loaded with trash, disconnected from the habitat, and stored on the surface. The total amount of logistics required over a human lunar scenario will dictate that a large number of carriers be delivered to the lunar outpost site. The crew will be required to unload, move, and connect each of these carriers. There is the potential that these actions could involve significant crew time, as well as increased risk to the crew. In addition, these issues could be compounded if required availability of logistics requires multiple movements of each carrier. A dynamic analysis of cargo usage and carrier positioning will allow for a prediction of required carrier manipulations and will allow for the development of logistics plans that minimize these actions.

- Availability of critical spares and maintenance items - Because most goods, including critical spares and maintenance items, will be delivered in carriers that will be stored on the surface, there is the possibility that not all items will be immediately available to the crew. With cargo being stockpiled on 
the surface and limited interfaces with the habitat, certain pressurized carriers will be stored on the surface without connection to the habitat. If a critical spare is on the surface, but located in a carrier that is not currently connected to the habitat, the crew would have to switch out carriers in order to gain access to those supplies. In a critical situation, this could increase the risk to the outpost and the crew. A dynamic analysis of carrier positioning allows for manifesting and logistics planning that maximizes the availability of critical spares.

- Availability of contingency consumables - As with critical spares, there is also a potential issue regarding the availability of contingency consumables. If there is a failure event that causes the crew to shelter in place in the habitat beyond the planned surface duration, extra consumables will be required. While these consumables are on the surface, they may be located in a carrier that is not connected to the habitat. In a contingency situation there may be no way for the crew to gain access to these supplies. A dynamic analysis of cargo positioning will allow for logistics planning that provides full access to contingency spares at all times.

- Storage duration for logistics - Because goods are delivered on a cargo carrier and stored on the surface for use by future crews, it is possible that some consumables may sit on the surface for extended periods of time. Goods that have a limited lifetime may expire before they are needed. A dynamic model of cargo storage and usage will allow for a manifesting plan that will allow for all goods to be consumed before they expire.

- Requirements for delivery of "as needed" items - Because the storage capability at the outpost and total delivery capability are limited, there will be a significant number of maintenance and repair items that will be delivered on an "as needed" basis. These are items that are not of high criticality (i.e. failure will not result in loss of mission or loss of crew) but could result in lowered availability of equipment or loss of productivity. A dynamic model of sparing requirements, delivery, and usage could help determine which types of spares should be pre-positioned and which should be delivered as needed. This type of sparing plan will then feed back into the delivery requirements for a scenario.

- Storage capacity for trash - Storage of trash presents a unique storage challenge for the lunar outpost. Because there is no capability to remove trash from the outpost, it must be permanently stored at the site. Contamination and preservation issues will require a robust containment of the trash. A dynamic analysis of trash storage requirements will allow for logistics planning that allows for full disposal of goods and will not result in a build-up of material in the habitat.

\section{Analysis Capabilities}

In order to properly evaluate micro-logistics constraints and to determine to what degree those constraints might limit ultimate productivity at a human lunar outpost, the NASA Constellation Program Strategic Analysis Team (SAT) developed a micro-logistics model that has the capability to track the delivery, positioning, usage, and disposal of all goods at an outpost site.

The micro-logistics model is comprised of two separate analysis modules and a visualization tool. The two analysis modules simulate micro-logistics at a human lunar outpost over time using a system dynamics methodology. One analysis module tracks the delivery, storage, and conversion of all liquids and gasses at the outpost site. The second module tracks the delivery, storage, usage, and disposal of all solid goods at the outpost. The visualization tool provides for interpretation and analysis of scenario results in an interactive manner. The system dynamic analysis modules were developed using iSee Systems iThink software ${ }^{3}$. The visualization module, which is directly linked to the analysis modules for data transfer, was developed using Microsoft Excel software with Visual Basic for Applications (VBA).

The micro-logistics model is run against a standardized scenario description, which is produced by the lunar exploration analysis tools ${ }^{4}$ employed by the SAT. The scenario description specifies the mission schedule, crew durations, and element delivery for a specific scenario to be evaluated. In addition, the scenario description captures the manifesting plan for each mission. The manifesting plan for a given scenario specifies the carriers loaded on each flight and the specific types and amount of cargo in each carrier. This plan is developed as part of the scenario definition process, using the manifesting tools developed by the SAT. The micro-logistics model imports the 
scenario description, and based on the input parameters produces a time-based simulation of all micro-logistics activities at the outpost.

\section{A. Gas/Liquid Micro-Logistics Module Description}

The gas/liquid module simulates the operations of the ECLSS system, including all storage location, processing stages, and human functions. Figure 1 shows the visual representation of the gas/liquid module.

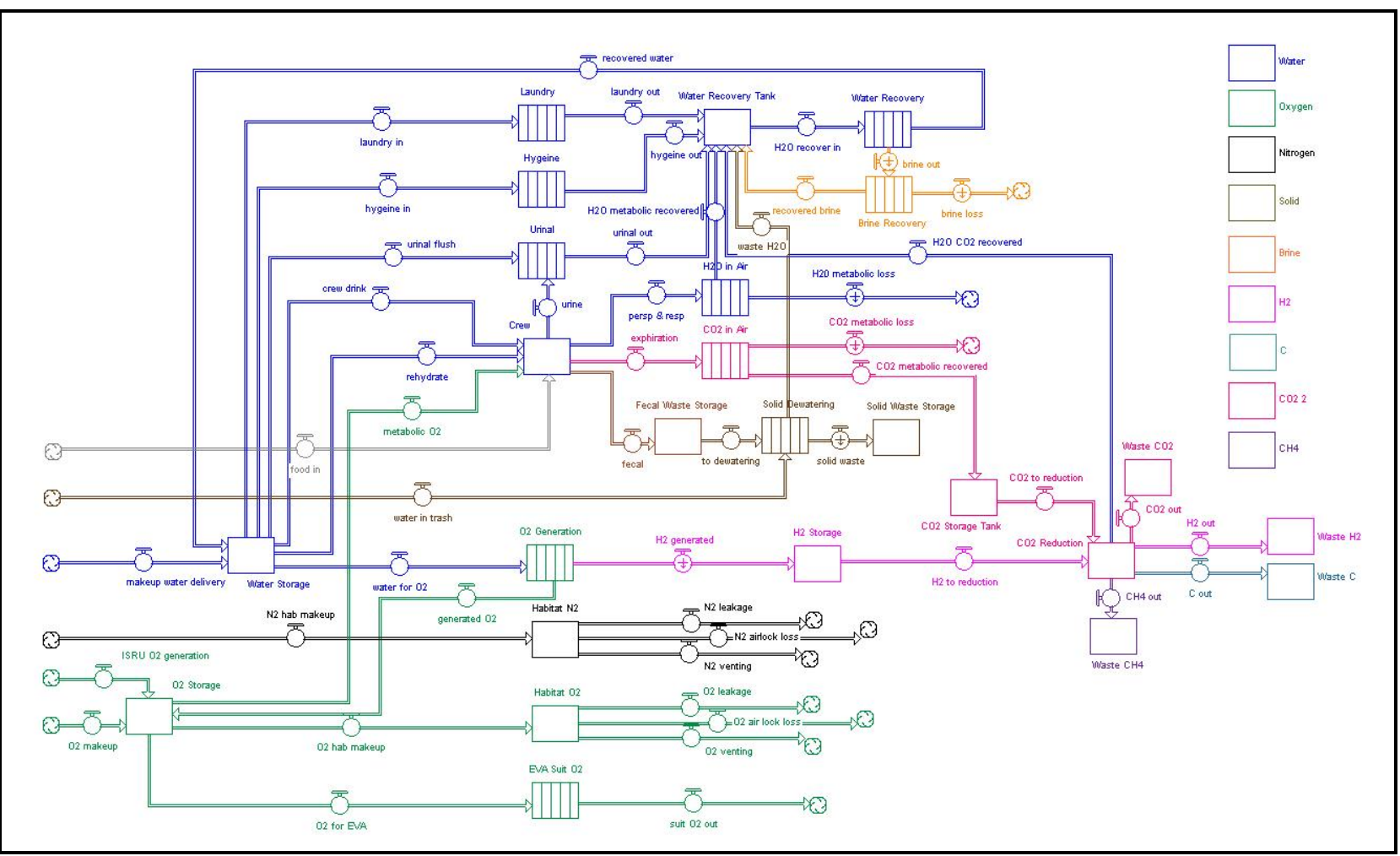

Figure 1: Gas/Liquid Micro-Logistics Module

In Figure 1, each separate type of gas or liquid in the ECLSS system is represented by a different color. Solid rectangles represent storage locations and hashed rectangles represent a process where a gas or liquid is converted in form, either as a process in the ECLSS system or as a human bodily function. Flows of gas or liquid from one location to another are represented as arrows. The system dynamics methodology in which the gas/liquid module is built ensures that mass is conserved. For each process in the model, the input and output rates for each gas or liquid type is specified and the total mass must balance. Specific types of gasses and liquids tracked in the module include water, oxygen, nitrogen, water in food, solid waste, brine, hydrogen, carbon, carbon dioxide, and methane.

The gas/liquid module is dynamic in nature. As the module simulates the operation of the ECLSS system over time, real usage schedules and rates for each process are used to drive the analysis. The crew at the outpost will interact with the ECLSS system based on their schedule of activities. Specific activities include:

- $\quad$ Sleeping

- Exercising

- $\quad$ Eating

- Drinking

- Personal hygiene

- Doing laundry

- Using the urinal

- Using the toilet

- Extra Vehicular Activity (EVA)

The schedule of activities drives the consumption and production rates for gas and liquids for each crewmember. For example, while sleeping, crewmembers breathe at a minimal rate. While exercising, they will breathe at an 
accelerated rate. While on EVA, the crew will not consume any oxygen from the habitat, but will consume a set amount of oxygen using the EVA system. During the rest of the activities, crewmembers will breathe at a normal rate. The model tracks these activities to calculate the total hourly oxygen usage rate, as well as the rate at which byproducts of breathing are produced.

Water, food, and solid waste are also used and produced in a stochastic manner. Water is used for drinking, food rehydration, hygiene, laundry and urinal flushing by the crew. In addition, water may be used in the ECLSS system for the generation of oxygen. The usage rate for water is based on the developed schedule for eating, hygiene, and laundry activities. Food (water in food) is consumed during several meal breaks scheduled throughout the day. Hygiene processes produce dirty water, carbon dioxide, urine and solid waste. The crew will release water into the air through perspiration and respiration and they will also release carbon dioxide through expiration. The model tracks the production and recovery of water and oxygen from the byproducts of the different crew activities. These recovered materials are then available for processing in the ECLSS system

Wastewater can be collected from laundry, hygiene, urinal, air filtering, carbon dioxide reduction and solid waste dewatering. At the end of each one of these processes the wastewater is collected in a water recovery tank to await purification. The purification process produces brine, part of which will be reintroduced into the water recovery tank for further extraction of water. The water purified through this process is added to the main water storage in the habitat.

\section{B. Solid Goods Micro-Logistics Model Description}

The solid goods module tracks the delivery, storage, usage, and disposal of solid goods at the lunar outpost. Figure 2 provides a visual presentation of the solid goods module.

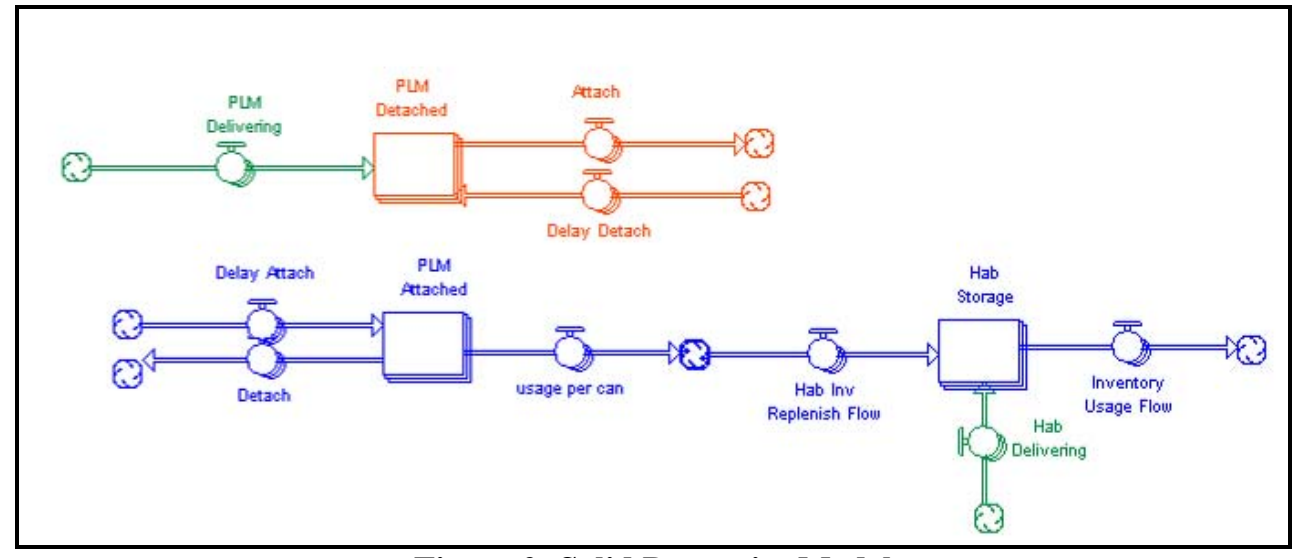

Figure 2: Solid Dynamics Model

As in the gas/liquid module, storage locations are represented by solid rectangles in Figure 2. Arrows represent transfer of goods from one location to another. For the solid goods model, the colors in Figure 2 represent the status for the goods. Goods are designated as being delivered, stockpiled away from the habitat, stockpiled at the habitat, or as trash. At each location, the inventory of goods is sub-divided by type. The following categories of goods are specifically tracked in the model:

- Food

- $\quad$ Crew consumables

- $\quad$ Crew effects

- $\quad$ Critical spares

- Maintenance items or other spares

- Science or utilization goods

The solid goods module primarily tracks the storage location for each type of good. Cargo storage within the habitat is limited and will be spread through the multiple habitat components. As the habitat components are delivered to the lunar surface and integrated to the habitat, more storage capacity will become available. The model tracks the delivery of carriers and calculates total habitat storage capacity to the system based integration schedule for the habitat components.

After the completion of the habitat integration, additional solid goods will be delivered in pressurized logistics carriers. These carriers, referred to as PLMs (Pressurized Logistics Modules), will be attached to the habitat, 
providing additional storage capacity. Cargo will be moved from the carriers into the habitat storage capacity to replenish needed materials. Trash is moved from the habitat into the carriers as space is made available. When a carrier is exhausted or nearly exhausted, it is exchanged for another pressurized carrier. Specific storage locations within a habitat or carrier are not modeled within the micro-logistics tools. It is assumed that a standard storage unit, such as Crew Transfer Bags (CTBs), similar to those used on ISS, will be used. Therefore, there will be no additional constraints in storing cargo in specific locations.

Consumption and conversion of materials is also specifically simulated in the model. As with the gas/liquid module, the schedule of crew activities drives the consumption rates. Food, crew consumables and effects, and science/utilization goods are consumed or converted to trash based upon the schedule of crew activities. Maintenance and repair requirements drive the use of spares. Critical spares will be used to repair unscheduled critical system failures. The failures are generated randomly, based upon expected mean time between failure (MTBF) data in the model. Maintenance spares are consumed based on the maintenance schedule for the habitat systems.

\section{Visualization of Results}

A third module was developed to visualize and evaluate the results of the micro-logistics analysis from both models. This tool was designed to visually represent the total quantity of goods at the lunar outpost site, broken down by logistics type and current location, at any given time. The visualization tool has the capability to depict each habitat elements and independent carrier that is on the surface and to show the breakdown of what goods are located in each. The visual representation is captured as a series of individual points in time over the life of the scenario. The tool can be queried to show the current logistics status at any given point of time or the entire history of a scenario can be shown as a movie.

Figure 3 shows a visual representation of the logistics of the lunar outpost at an early stage of outpost build-up. Figure 4 shows the same representation at a much later point in time when the outpost is operating with continuous human habitation.

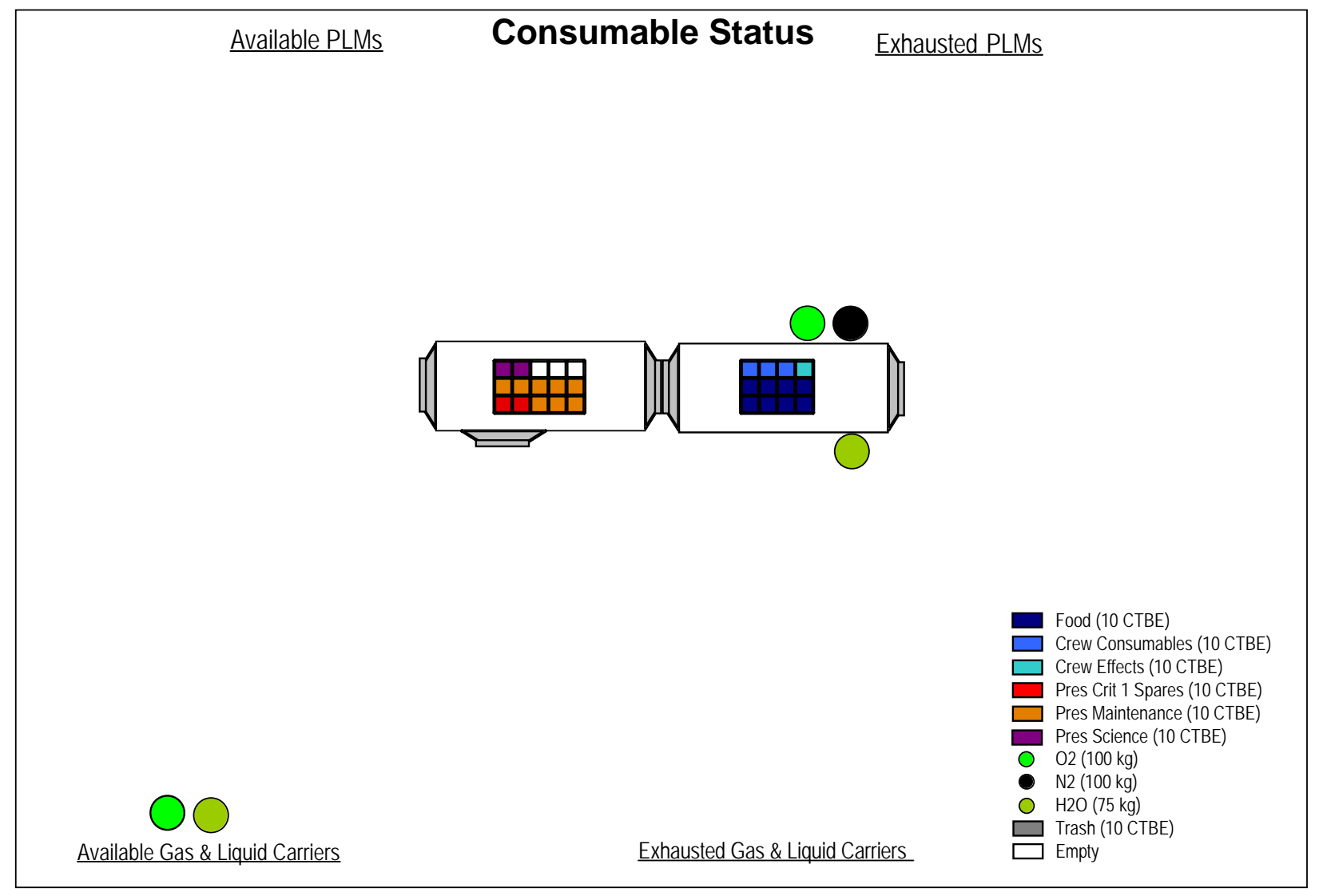

Figure 3: Visualization of Micro-Logistics for Early Outpost Mission 
In Figures 3 and 4 solid goods are represented as "packets", with each packet equivalent to 10 Cargo Transfer Bag Equivalents (CTBE). CTBE are a standard volumetric unit of measure for pressurized resupply goods. Each packet is represented as a single square in the diagrams. The color of the square represents the class of goods represented by each packet. Blank squares represent unfilled storage capacity in each element. In addition, each packet is "tagged" with information regarding its history. This information allows an analyst to determine how long each packet has been on the surface, when and how it was delivered, and when it was transferred.

Liquids and gasses are represented as numbers of full or fractionally full carriers. A carrier for each type of gas or liquid has a standard volumetric capacity. The model shows the location of each carrier and how full each is at any given point in time. The color of the carrier indicates the type of liquid or gas that is contained.

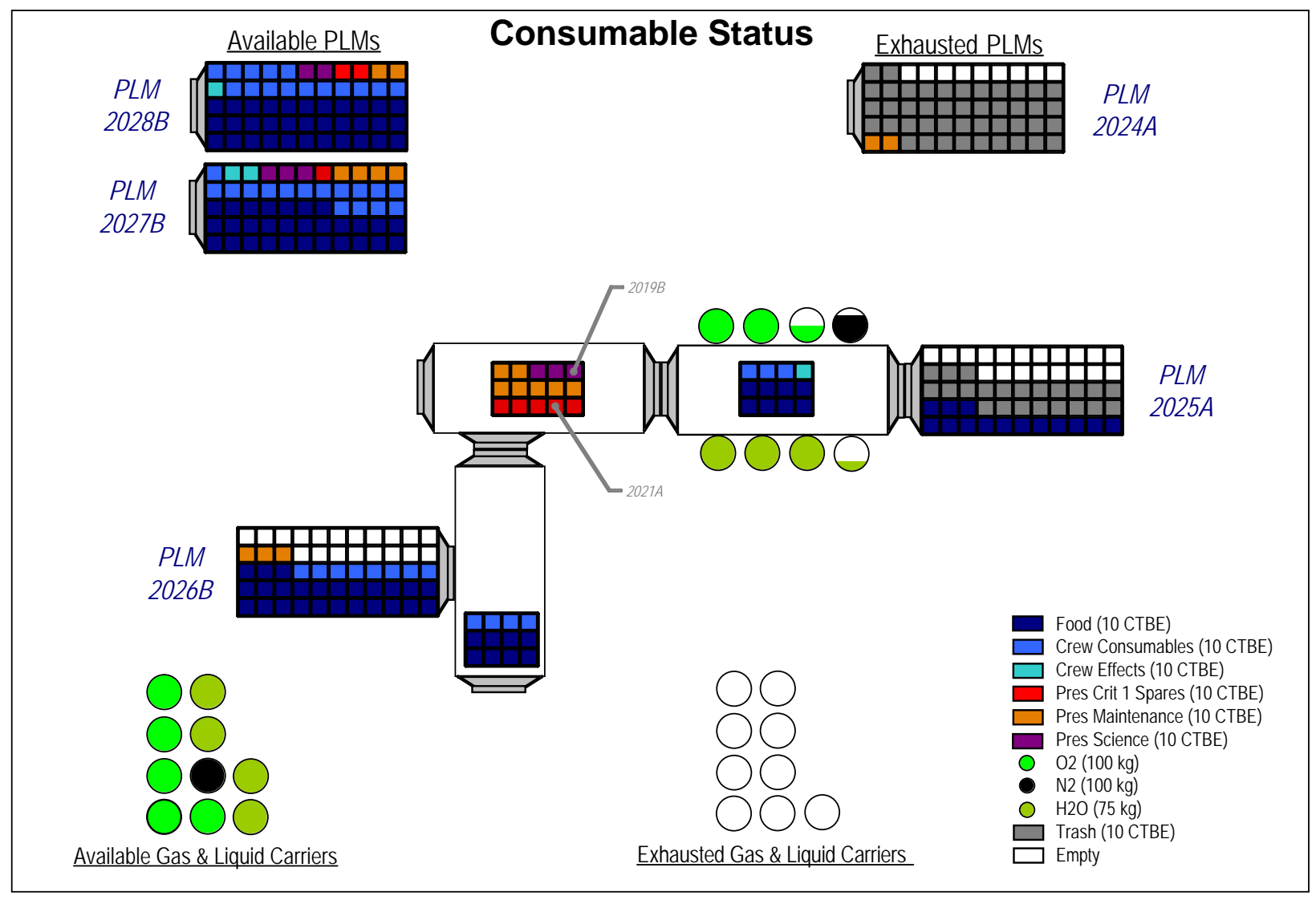

Figure 4: Visualization of Micro-Logistics for Later Outpost Mission

Using the visualization module, it is possible to evaluate the micro-logistics performance of a given scenario and to evaluate the specific challenges outlined in Section III of this paper. The results of the micro-logistics analysis will indicate whether the current habitat volume, in conjunction with the current logistics plan, will allow for carriers to be completely unloaded and will also allow for the storage of trash without impacting habitable volume. The results will also indicate the availability of critical spares and contingency consumables and the storage duration for all life-limited goods. If the results are not acceptable in any of these areas, the habitat volume could be increased or, more commonly, the logistics plan that was developed for the scenario can be modified. The micro-logistics results will indicate which carrier could not be fully unloaded, the time period in which there were volumetric constraints, and when there was a build-up of trash. This data allows analysts to modify the loading of carriers to correct these issues. In addition, the micro-logistics module presents a summary of the total number of carrier movements required for a scenario and the crew time associated with those movements. If the number of movements is found to be excessive, the logistics plan may be similarly modified.

American Institute of Aeronautics and Astronautics 092407 


\section{Summary}

The constraints imposed due to micro-logistics considerations will impose limitations on the usage and productivity of a human lunar outpost. Innovative types of logistics analysis and planning will need to be conducted in order to develop manifesting and logistics plans that result in an acceptable level of overall system performance. The Strategic Analysis Team has successfully developed a new tool to evaluate micro-logistics constraints for human lunar scenarios and has integrated this analysis into the overall strategic analysis process. The result is that constraints from the local handling of logistics are now fully evaluated and those limitations and requirements are used to design outpost elements, to develop practical logistics plans, and to produce viable, robust exploration scenarios.

\section{References}

\footnotetext{
${ }^{1}$ Andraschko, M., Merrill, R. G., Earle, K., 2008, “Logistics Modeling for Lunar Exploration Systems,” AIAA Space 2008 Conference \& Exposition, San Diego, California, 2008 (to be published).

${ }^{2}$ Stromgren, C., Andraschko, M., Merrill, R. G., Cirillo, W., Earle, K., Goodliff, K., “A Comparison of Probabilistic and Deterministic Strategic Analysis for Human Space Exploration,” AIAA SPACE 2008 Conference \& Exposition, San Diego, California, 2008 (to be published).

3iThink, Software Package, Ver. 9.0.2, iSee Systems, Inc., Lebanon, NH, 2006.

${ }^{4}$ Cirillo, W., Goodliff, K., Earle, K., Reeves, J.D., Stromgren, C., Andraschko, M., Merrill, R.G., "Strategic Analysis Overview,” AIAA Space 2008 Conference \& Exposition, San Diego, California, 2008 (to be published).
} 